\title{
BMJ Open Integrated versus nOn-integrated Peripheral inTravenous catheter. Which Is the most effective systeM for peripheral intravenoUs catheter Management? (The OPTIMUM study): a randomised controlled trial protocol
}

\author{
Maria Isabel Castillo, ${ }^{1}$ Emily Larsen, ${ }^{1,2}$ Marie Cooke, ${ }^{1}$ Nicole M Marsh, ${ }^{1,2}$ \\ Marianne C Wallis, ${ }^{1,3}$ Julie Finucane, ${ }^{1,4}$ Peter Brown, ${ }^{1,4}$ Gabor Mihala, ${ }^{1,5}$ \\ Peter J Carr, ${ }^{1,6}$ Joshua Byrnes, ${ }^{5}$ Rachel Walker, ${ }^{1,7}$ Prudence Cable, ${ }^{4}$ Li Zhang, ${ }^{1}$ \\ Candi Sear, ${ }^{1,4}$ Gavin Jackson, ${ }^{1,6}$ Anna Rowsome, ${ }^{1,4}$ Alison Ryan, ${ }^{4}$ \\ Julie C Humphries, ${ }^{4}$ Susan Sivyer, ${ }^{4}$ Kathy Flanigan, ${ }^{4}$ Claire M Rickard ${ }^{1,2,7}$
}

To cite: Castillo MI, Larsen E, Cooke $\mathrm{M}$, et al. Integrated versus nOn-integrated Peripheral inTravenous catheter. Which is the most effective systeM for peripheral intravenoUs catheter Management? (The OPTIMUM study): a randomised controlled trial protocol. BMJ Open 2018;8:e019916. doi:10.1136/ bmjopen-2017-019916

- Prepublication history for this paper is available online. To view these files, please visit the journal online (http://dx.doi. org/10.1136/bmjopen-2017019916).

Received 8 0ctober 2017 Revised 27 February 2018 Accepted 5 April 2018

Check for updates

For numbered affiliations see end of article.

Correspondence to

Ms. Emily Larsen;

emily.larsen@health.qld.gov.au

\section{ABSTRACT}

Introduction Peripheral intravenous catheters (PIVCs) are frequently used in hospitals. However, PIVC complications are common, with failures leading to treatment delays additional procedures, patient pain and discomfort, increased clinician workload and substantially increased healthcare costs. Recent evidence suggests integrated PIVC systems may be more effective than traditional non-integrated PIVC systems in reducing phlebitis, infiltration and costs and increasing functional dwell time. The study aim is to determine the efficacy, cost-utility and acceptability to patients and professionals of an integrated PIVC system compared with a non-integrated PIVC system.

Methods and analysis Two-arm, multicentre, randomised controlled superiority trial of integrated versus nonintegrated PIVC systems to compare effectiveness on clinical and economic outcomes. Recruitment of 1560 patients over 2 years, with randomisation by a centralised service ensuring allocation concealment. Primary outcomes: catheter failure (composite endpoint) for reasons of: occlusion, infiltration/extravasation, phlebitis/ thrombophlebitis, dislodgement, localised or catheterassociated bloodstream infections. Secondary outcomes: first time insertion success, types of PIVC failure, device colonisation, insertion pain, functional dwell time, adverse events, mortality, cost-utility and consumer acceptability. One PIVC per patient will be included, with intentionto-treat analysis. Baseline group comparisons will be made for potentially clinically important confounders. The proportional hazards assumption will be checked, and Cox regression will test the effect of group, patient, device and clinical variables on failure. An as-treated analysis will assess the effect of protocol violations. Kaplan-Meier survival curves with log-rank tests will compare failure by group over time. Secondary endpoints will be compared between groups using parametric/non-parametric techniques.
Strengths and limitations of this study

This is a large-scale, multicentre randomised controlled trial (RCT) of superiority design to investigate the efficacy and cost-utility of an integrated peripheral intravenous catheter (PIVC) system in adult medical/surgical patients to prevent PIVC complications.

- This pragmatic RCT will involve PIVCs inserted and cared for by clinicians in three hospitals using existing protocols, not specialist teams or researchers and as such will provide findings applicable to the majority of patients with PIVCs.

- Microbiology endpoints will be analysed by blinded scientists, and infection outcomes will be assigned by a blinded infectious disease physician assessor.

- The PIVC system cannot be blinded to clinical staff, patients or research nurses.

- Children ( $<18$ years old) are excluded from the study, so results will not be generalisable to this group.

Ethics and dissemination Ethical approval from the Royal Brisbane and Women's Hospital Human Research Ethics Committee (HREC/16/QRBW/527), Griffith University Human Research Ethics Committee (Ref No. 2017/002) and the South Metropolitan Health Services Human Research Ethics Committee (Ref No. 2016-239). Results will be published in peer-reviewed journals.

Trial registration number ACTRN12617000089336.

\section{INTRODUCTION}

Peripheral intravenous catheters (PIVCs) are frequently used to provide treatment for hospital patients. ${ }^{1}$ However, the failure rate of these devices is extremely high with up to $69 \%$ 
failing before end of treatment. ${ }^{2}$ Common complications leading to catheter failure include phlebitis (sometimes called thrombophlebitis), occlusion, dislodgement, infiltration (including extravasation), and less often, localised or bloodstream infections. ${ }^{34}$ A recent randomised controlled trial (RCT) of 642 patients in Spain suggests that integrated PIVC systems-preassembled systems containing the intravenous catheter and extension tubing incorporated as one piece, and with a flatter hub profile resting against the skin surface-are superior to traditional non-integrated PIVC systems (where separate components need to be attached, with a rounded hub profile against the skin). ${ }^{5}$ That study reported that the use of integrated PIVCs reduced phlebitis by a relative $29 \%$ (31 vs 45 cases/1000 catheter-days; $\mathrm{p}=0.004$ ), increased the functional dwell time (median 144.5 hours (95\% CI) 123.4 to 165.6 ) vs 99 hours (95\% CI 87.2 to 110.8 ), $\mathrm{p}<0.001$ ) among PIVCs in place for $\geq 24$ hours and substantially reduced associated costs (€786 257/ year/1000 beds when PIVCs replaced based on clinical indication). ${ }^{5}$ However, to our knowledge, this is the only published RCT of this new technology, and it remains to be tested in other healthcare systems.

Integrated PIVC systems have recently been introduced into clinical practice and are designed to facilitate longer PIVC dwells that are now possible since a worldwide reassessment of routine 48-96 hour removal policies for PIVCs. ${ }^{367}$ Since 2011, major guidelines have begun to recommend only clinically indicated removals of PIVCs, that is, removal on completion of therapy or, for complications such as phlebitis, infiltration, occlusion, accidental removal or suspected infection. ${ }^{8}$ Replacing catheters when clinically indicated, compared with replacement on a specific time schedule, significantly reduces healthcare costs and patient discomfort. However, PIVC failure remains highly prevalent; hence, new technologies such as the integrated PIVC system need to be tested and, if effective and cost-effective, consistently implemented. ${ }^{35}$

Integrated PIVC systems may reduce internal pressures on the vein incurred when staff manipulate the catheter to connect the extension set. Postinsertion, the use of integrated catheters means that additional fluid tubing connection or direct injections are undertaken via the dedicated port, slightly upstream of the catheter itself, again likely reducing movement of the catheter body against the internal vein wall. Less movement may therefore be key in reducing irritation of the tunica intima of the vessels, which in turn decreases vessel swelling and consequently painful oedema, occlusion or infiltration. The design may also reduce the risk of contamination and infection by reducing disconnection and manipulation, as well as accidental disconnection since the extension tubing is a built-in feature. ${ }^{9}$ These catheters have a flat/rectangular face on the surface placed against the skin and a round/ half-cylindrical shape at the top. It is possible that, compared with traditional fully cylindrical-shaped catheters, this design reduces external pressure on the vein, causing less irritation and subsequent phlebitis, internal swelling and occlusion.

Since PIVC complications and failure lead to delays in treatments, additional procedures, patient pain and discomfort, increased work for clinicians and increased healthcare cost, it is imperative to find new methods of preventing these negative outcomes. Despite the promising results reported by González López et a $a \tilde{l}$ with integrated system PIVCs, replication of their findings in other clinical settings and patient populations is needed to provide stronger evidence to inform clinical practice and revised guidelines.

\section{Objectives}

The aim of the study is to determine the efficacy, costutility and acceptability to patients and professionals of the integrated PIVC system versus non-integrated PIVC systems to assist policy makers with decision making about the best PIVC system choice for patients. The objectives are:

1. To compare the clinical and cost-utility of PIVC integrated systems and non-integrated catheter for the prevention of PIVC failure, insertion pain, catheter-associated bloodstream infection (CABSI), functional dwell time and adverse events.

2. To assess the acceptability and challenges of implementation of the integrated system via quantitative and qualitative feedback from inserters, patients, clinical nurses and policy makers.

\section{METHODS AND ANALYSIS \\ Trial design}

The study is a two-arm (table 1), multicentre RCT of superiority design comparing two PIVC systems to prevent PIVC complications and failure. In addition, covariates associated with catheter failure including patient variables and catheter variables will be evaluated.

\section{Hypothesis}

The use of an integrated PIVC will reduce the incidence of PIVC failure compared with the use of a non-integrated PIVC.

\begin{tabular}{ll}
\hline Table 1 & Study arms \\
\hline Study arms & Catheter type \\
\hline $\begin{array}{l}\text { Integrated } \\
\text { system }\end{array}$ & $\begin{array}{l}\text { BD NexivaTM Closed IV Catheter System } \\
\text { Dual Port with SmartSite needleless } \\
\text { connectors (BD, Utah, USA), } \mathrm{n}=780 \\
\text { patients. }\end{array}$ \\
$\begin{array}{l}\text { Non-integrated } \\
\text { system }\end{array}$ & $\begin{array}{l}\text { B Braun Introcan Safety 3 Catheter (B } \\
\text { extension sets and needleless connectors } \\
\text { will be used as per standard site practice, } \\
\mathrm{n}=780 \text { patients. }\end{array}$ \\
\hline
\end{tabular}

Standard care products may change within the facilities during this trial and this will be controlled for as a potential covariate. 


\section{Sample size and study power}

For the sample size calculation, we hypothesised a relative reduction in PIVC failure of 0.83 to be associated with integrated PIVCs as reported in previous research. ${ }^{5}$ Based on a known local baseline PIVC failure incidence of $41 \%$ (Marsh N. Peripheral Venous Catheter Dressing and Securement: Results from a 4-group Randomized Controlled Trial of 1709 Patients in 2 Hospitals. Association for Vascular Access Annual Scientific Meeting Dallas, Texas 2015), we anticipate an observed failure incidence of $34 \%$ in the integrated PIVCs. With $80 \%$ power, $5 \%$ alpha and additional $2.5 \%$ for potential attrition, we estimate a total required sample size of 1560 patients (780 integrated and 780 non-integrated). ${ }^{10}$

To ensure safety, maximise resources and reduce unnecessary enrolment of patients, a blinded primary endpoint analysis will be undertaken when $50 \%$ of the sample (780 participants) have been recruited. An Independent Data Safety and Monitoring Committee will review the analysis and adverse event data then provide advice to the research team on sample size, adjustments or study stopping. This advice will be reviewed by RW, EL, MC, NMM and CMR, who will make the final decision to terminate the trial.

\section{Setting and sample}

The trial will be undertaken at three Queensland, Australian metropolitan hospitals: The Royal Brisbane and Women's Hospital, The Queen Elizabeth II Jubilee Hospital and the Princess Alexandra Hospital. Study sites will commence recruitment sequentially to allow adequate time for local refinement of study processes and support from the project manager. Prospective patients scheduled to have a PIVC inserted will be invited to participate. Patients who meet the following inclusion criteria and no exclusion criteria (table 2) will be eligible.

\section{Outcome measures and definitions}

Primary outcome

The primary outcome is PIVC failure.

1. PIVC failure: catheter failure (composite endpoint) for reasons of: occlusion, infiltration/extravasation, phlebitis/thrombophlebitis, dislodgement, localised or bloodstream catheter-related infections.

a. Occlusion defined as the loss of the ability to infuse fluids and/or medications through a previously functioning PIVC. ${ }^{11}$

b. Infiltration/extravasation: infiltration is defined as the inadvertent permeation of intravenous fluid (non-vesicant solution) into the interstitial compartment, causing swelling of the tissue around the site of the catheter. Extravasation is the inadvertent administration of a vesicant solution into surrounding tissue. ${ }^{12} 13$

c. Phlebitis/thrombophlebitis is defined as irritation and inflammation of a vein wall caused by the presence of the PIVC. It is characterised by the presence of any combination of tenderness, pain, erythema, swelling, warmth, palpable cord or pu-

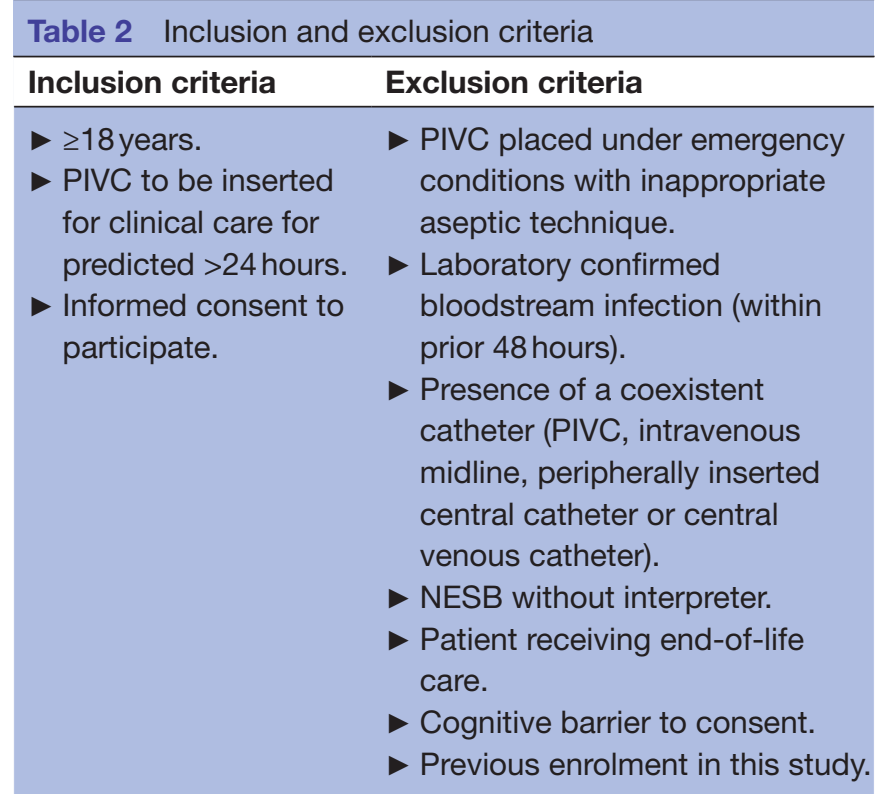

NESB, non-English-speaking background; PIVC, peripheral intravenous catheter.

rulent drainage. ${ }^{14}$ In this study, phlebitis/thrombophlebitis includes pain (>1 out of 10) alone or plus any of the criteria mentioned above (on questioning, then palpation by the research nurse $(\operatorname{ReN}))$.

d. Dislodgement is defined as movement of the catheter out of the vein resulting in partial or complete dislodgement. ${ }^{14}$ This may be characterised by leaking (partial dislodgement).

e. Localised venous infection (without bloodstream infection) using the CVS-VASC criteria of the Centers for Disease Control and Prevention (CDC) National Healthcare Safety Network. This is defined as purulent drainage from the PIVC insertion site or symptoms of infection at the site (eg, pain, erythema) with $>15$ colonies cultured from the PIVC tip. ${ }^{15}$

f. CABSI using the BSI-LCBI criteria of the CDC, ${ }^{15}$ confirmed by a blinded infectious disease specialist using deidentified data. This is defined as a laboratory confirmed BSI (recognised pathogen or two matching common commensals within 2 calendar days) and at least one symptom of infection (eg, fever $>38.0^{\circ} \mathrm{C}$ or hypotension) which is not related to an infection at another site.

\section{Secondary outcomes}

Failure type (as above); first time insertion success; device colonisation ( $>15$ colony-forming units $(\mathrm{cfu})$ growth on tip after removal); insertion pain (Numeric Rating Scale: $0=$ no pain to $10=$ extreme pain); functional dwell time; adverse events; mortality; direct hospital costs; health-related quality of life utility score; and patient and clinician satisfaction. 


\section{Recruitment, randomisation, allocation concealment and} blinding

Full-time ReNs will screen patients, gain informed consent from $\sim 48$ patients per week ( 16 per site), educate clinical staff, patients and families, monitor protocol compliance and collect daily patient data 'on trial' (ie, until removal of device). After consent is gained, ReNs will randomise each of the 1560 patients ( $\sim 520$ at each site) via a central web-based service (Griffith University) ${ }^{16}$ with randomly varied block sizes to ensure allocation concealment until study entry. Randomisation will be a 1:1 ratio (integrated:non-integrated) between groups, stratified by study site. Data will be recorded using REDCap software (Research Electronic Data CAPture, Vanderbilt) ${ }^{17}$ on password-protected hand-held devices. It is not possible to blind patients and clinical staff because of the nature of the intervention. However, the microbiologist, infectious disease physician and statistician will be blinded.

\section{Interventions}

Insertion and care of the PIVCs

Study and control catheters will be inserted by trained clinicians (either clinical staff or ReNs) at each hospital, who are existing skilled or competent intravenous inserters. Pretrial they will have training and simulated practice inserting the study catheter, until they feel confident that their skills match their competence for control catheter insertion. If ultrasound is used to guide insertion, this will be recorded.

Patients will have one PIVC entered into the study so that the unit of analysis (the patient) is independent. Furthermore, PIVCs can fail at any time of day and need to be replaced quickly; it would therefore not be possible to have subsequent insertions for participating patients to continue in the study due to training deficit of general hospital staff in integrated PIVC insertion. If patients have a failed PIVC, they will receive the standard institutional PIVC inserted by the hospital staff. Patients will have other aspects of PIVC care (eg, skin preparation and dressings) standardised in all study groups, consistent with local policy and practice, although some variation is expected between staff practices as this is a pragmatic trial.

\section{Data collection}

We will collect quantitative and qualitative data from patients, PIVC inserters, clinical nurses and policy makers.

\section{Preparation phase}

Data will be collected on:

A. The training of clinicians: about 20 clinicians (nurses, anaesthetists and/or emergency clinicians) who are current specialist or competent inserters (ie, who regularly insert at least five cannulas per week) will be trained using the integrated PIVC system. During training, we will record:

- Clinicians' level of satisfaction with the integrated system: inserting, accessing (eg, giving meds/ flush), securing and dressing ( $0=$ noteasy to $10=$ very easy).

- The number of simulated insertions, and/or clinical insertions, for clinicians to feel $>80 \%$ and $>90 \%$ confident to achieve first time insertion success, and if first time insertion was achieved.

- Feedback from clinicians about the process of developing this skill using 'think-aloud' interviews, ${ }^{18}$ which will be audio-taped, transcribed and analysed thematically.

\section{Clinical use phase}

Data will be collected on:

A. Patient characteristics (baseline) collected will include: age, gender, premorbid history, reason for hospital admission and presence of infection. PIVC-related information collected are: type (brand), gauge, insertion site, side of insertion, clinical area, inserter discipline, vein quality, number of insertion attempts, first/subsequent PIVC, use of ultrasound and additional attachments (eg, needleless connectors and extension tubing). Data collected during daily assessment will include: dressings and securements in place, phlebitis signs and symptoms, infusates given and additional attachments. Following device removal, data gathered will include: reason for catheter removal, catheter-related complications, dwell time, infusates, subsequent PIVC (yes/no), presence of infection (including bloodstream infection), PIVC sample cultures results (if ordered by the treating team) and mortality. Patients' pathology results will be followed for 48 hours after the removal of the PIVC to assess infection and adverse events. Consistency of PIVC site assessment and data entry will be safeguarded by the use of: extensive education prior to trial commencement; education at regular intervals; and the provision of a standardised handbook of standard operating procedures).

B. Patient PIVC indwell experience:

- Health-related quality of life survey: EuroQol 5-dimension 5-level questionnaire (EQ-5D-5L) ${ }^{19}$ $(\mathrm{n}=350$ between the three sites, with an interim analysis at $n=200)$. This instrument will be administered twice: (1) prior to the insertion of the PIVC (baseline) and (2) close to the expected end of treatment (24-60 hours after PIVC insertion).

- Patient satisfaction survey: Functional Assessment of Chronic Illness Therapy - Treatment Satisfaction - General $^{20}(\mathrm{n}=350$ with an interim analysis at $n=200)$. This instrument will be administered once: (1) close to the expected end of treatment (24-60 hours after PIVC insertion).

- Positive and negative feedback provided by patients will be collected by the ReN as field notes and analysed thematically.

C. Clinician's level of satisfaction with the integrated and non-integrated systems: 
- Clinician satisfaction survey: reported ease of accessing (eg, giving meds/flush), securing, dressing and overall care $(0=$ not easy to $10=$ very easy).

- ReNs will also document field notes of any feedback provided by clinicians responsible for inserting, maintaining and/or removing PIVCs. Comments will be analysed thematically. ${ }^{21}$

D. Policy drivers and decision makers' views on the barriers and enablers of decisions about PIVC policy and potential implementation of integrated system PIVCs:

- Focus groups/interviews: once the clinical trial results are available, small focus groups will be held with local decision makers and policy drivers who choose types of catheter for the institution (ie, from departments of vascular access, infection prevention, purchasing, anaesthesia and emergency). Focus group attendees will be invited purposively from each department after consultation with directors and managers of each area regarding available and suitable candidates. A letter of invitation will be sent via email with each invitee asked to nominate a proxy if they cannot attend. Alternatively, a one-on-one interview will be scheduled. Some, but not all attendees, will have had experience of using the catheter. The results of the trial and clinicians' feedback will be presented, and semistructured questions will be asked of the group such as: 'How are we currently making decisions as to which catheter to use?'. They will also discuss how to respond to results. A moderator will guide the conversation to reveal potential barriers and enablers to implementation. A second researcher will note group dynamics and non-verbal behaviours. The data will be audio-taped, transcribed, coded, categorised and analysed thematically following the Norwood framework. ${ }^{21}$

\section{Cost-utility analysis}

A within-trial, cost-utility analysis will be conducted from the perspective of the hospital. It will estimate any potential incremental gain in utility measured in quality-adjusted life years (QALY) (based on survival and completed EQ-5D-3L surveys) as well as the incremental change in costs. The analysis will compare the local purchase costs of the combined integrated system, with the cost of the non-integrated system (and the additional attachments required). Detailed resource use for PIVC insertion, plus dressing and securement application, will be recorded for up to 30 procedures per inserter (convenience sample). Staff wage costs for device insertion, consultation and equipment used will also be recorded and included. The primary outcome will be the incremental cost-utility ratio (ICUR) with incremental costs, benefits and net monetary benefits also reported. Uncertainty in the ICUR estimates will be assessed using a probabilistic sensitivity analysis with $95 \%$ CI being reported along with one-way sensitivity analyses. The ICUR will be compared against a threshold willingness to pay value of $\$ 50000$ per QALY (a commonly used threshold in Australian cost-utility studies) with the sensitivity to decision making of this threshold value assessed using a cost-effectiveness acceptability analysis.

\section{Microbiological substudy}

Approximately 468 (234 per group) PIVC tips will be collected and analysed by the semiquantitative culture method $^{22}$ in the microbiological laboratory, Griffith University. PIVC tip selection will be based on availability of the ReN when the PIVC is removed and when transfer to the laboratory is available. When the PIVC is no longer required, the nursing staff will remove the PIVC. Qualified registered nurses with experience in preparation of specimens for culture will take the PIVC samples. The distal $2 \mathrm{~cm}$ of the tip will be removed using sterile scissors and deposited in a sterile container. All PIVC tips will be handled under aseptic conditions and immediately transported to a $4^{\circ}$ storage unit for later transfer to the laboratory for examination. Colonisation of PIVC tip will be considered at $>15 \mathrm{cfu}$. Results will be compared between groups with adjustment for relevant factors such as dwell time, body site of insertion, the clinical department where insertion occurred and antibiotic use. Blood cultures (if ordered by clinicians) from a peripheral vein will be cultured by Microbiology Pathology Queensland Central Laboratory.

\section{Patient and public involvement}

Anecdotal patient feedback, provided during the conduct of a large multicentre PIVC dressing RCT, undertaken in the same hospitals and health services, ${ }^{4}$ informed the overall design concept. Patients often reported feeling that the device and connections impacted on overall device function, success or failure. However, patients were not directly involved in the design of this study. While patients are required to provide informed consent to participate in the study, further study involvement in the health-related quality of life survey and patient satisfaction survey is completely voluntary and patients may refuse to participate regardless of their consent to participate in the main clinical study. Patient preferences are typically considered during the insertion of the PIVC; however, the final insertion site and device size may be influenced by clinical needs and physical limitations. Clinical staff, based on the wards, are responsible for all decision making in relation to the patient's PIVC care/maintenance and removal postinsertion; however, they are encouraged to include patient's preferences and concerns in this decision making. Trial participants are asked to indicate on the signed participant information and consent form whether they would like to receive a copy of the results on study completion. This will be sent by mail or email, depending on the preferred method selected by the patient. The burden of the intervention will be evaluated with the collection of field notes, recorded by the ReN. Patients will be asked to offer any positive and/ or negative feedback relating to trial processes, products and overall experiences. Specific patient advisers were not consulted for this study. 


\section{Statistical rationale and analysis plan}

Data will be cleaned and checked for missing and invalid values before importation into Stata. ${ }^{23}$ Descriptive characteristics and survey results will be presented using means and SD or medians and IQRs for continuous variables and counts/percentages for categorical variables. All randomised patients will be analysed by intention-totreat with patients as the unit of measurement, and only one PIVC analysed per patient. Baseline group comparisons will be by study group using clinical criteria to assess success of randomisation in distributing potential confounders between groups. Incidence rates of complications per 100 PIVC hours and incident rate ratios (with 95\% CIs) will be calculated. Kaplan-Meier survival curves will be presented to compare group failure in relation to dwell time, and the log-rank test will be used to compare the survival curves between study groups. A Cox regression will test the effect of group on failure, with hazard ratios calculated and adjustment for significant patient, device and clinical variables. Missing data will not be imputed. An as-treated analysis will assess the effect of protocol violations. $\mathrm{P}$ values $<0.05$ will be statistically significant. Secondary endpoints will be compared between groups using parametric/non-parametric techniques, as appropriate. A subgroup analysis will be undertaken to assess the effect of study site variability, with adjustments made in the main model should a significant effect be detected. The analyst will be blinded.

\section{DISCUSSION}

The current rate of PIVC failure is unacceptably high in hospitalised patients. PIVC failure results in negative patient-related outcomes, including increased pain and anxiety, delays in treatment and unnecessary exposure to the risks associated with repeated reinsertions. It also increases the work for clinicians and wastes millions of health dollars annually. Integrated PIVC systems are believed to be superior to the non-integrated PIVC systems in reducing catheter-related complications and cost. However, there is inadequate data to resolve uncertainty about their efficacy or safety compared with the non-integrated systems, as only one RCT has been published to date. At present, practitioners and policy makers make decisions with uncertainty due to lack of adequate evidence. This multicentre RCT will help to resolve uncertainty and inform international policy and practice.

\section{ETHICS AND DISSEMINATION}

The trial has been registered prospectively with the Australian New Zealand Clinical Trials Registry (ACTRN12617000089336). Approval was provided by the Royal Brisbane and Women's Hospital Human Research Ethics Committee (HREC/16/QRBW/527), Griffith University Human Research Ethics Committee (Ref No. 2017/002) and the South Metropolitan Health Services Human Research Ethics Committee (Ref No.
2016-239). Written informed consent to participate will be obtained from participants. Consent can be later withdrawn. Identifying details will be kept confidential via assigned numeric study IDs. The classification of adverse events will be graded using the Common Terminology Criteria for Adverse Events for reported adverse events. ${ }^{24}$ Serious adverse events will be monitored and reported to the Human Research Ethics Committees (HRECs), as will any important protocol modifications. If important protocol amendments are made (eg, changes to eligibility criteria), the principal investigator will update all investigators, HRECs, patient information and consent forms and the trial registry. Minor adverse events (eg, skin reaction to dressing) will be treated as per routine clinical practice with no cost to patients. Clinical trial insurance is held by the sponsor, Griffith University. A Data Safety and Monitoring Committee will review blinded interim data and adverse events at $\mathrm{n}=780$ to advise on safety. Annual reports will be provided to the HRECs. Before qualitative interviews and video recordings, participants will be asked to provide informed written consent. CMR, EL, NMM, GM, RW will have access to the final trial dataset. The trial and substudies will be written by the investigators and published in peer-reviewed journals, consistent with ICMJE Guidelines and authorship criteria. ${ }^{25}$

\section{Author affiliations}

${ }^{1}$ Alliance for Vascular Access Teaching and Research (AVATAR), Menzies Health Institute Queensland, Griffith University, Brisbane, Queensland, Australia

${ }^{2}$ Nursing and Midwifery Research Centre, Royal Brisbane and Women's Hospital, Brisbane, Queensland, Australia

${ }^{3}$ School of Nursing, Midwifery and Paramedicine, University of Sunshine Coast, Sunshine Coast, Queensland, Australia

${ }^{4}$ Queen Elizabeth II Jubilee Hospital, Brisbane, Queensland, Australia

${ }^{5}$ Centre for Applied Health Economics, Menzies Health Institute Queensland, Griffith University, Brisbane, Queensland, Australia

${ }^{6}$ Fiona Stanley Hospital, Murdoch, Western Australia, Australia

${ }^{7}$ Princess Alexandra Hospital, Brisbane, Queensland, Australia

Acknowledgements We thank Professor Tony Celenza and registered nurse Jodie Genzel for their input on the design of the project.

Contributors MIC, EL, MC, NMM, MCW, PJJC, GJ and CMR conceived the study. MIC, EL, MC, NMM, MCW, GM, PJJC, JB, RW, LZ, GJ and CMR designed the protocol. MIC, MC, MCW, NMM, PJJC, LZ, GJ and CMR secured funding. MIC wrote the first draft of the manuscript. EL wrote the final version of the manuscript. All authors contributed to and approved the final version of the manuscript.

Funding This work is supported by a Becton, Dickinson and Company (BD), Researcher Initiated Clinical Research Grant Scheme. This grant has been awarded with unrestricted conditions. The Sponsor is Griffith University (Australia). Additional in-kind support is gratefully received from Griffith University, the Royal Brisbane and Women's Hospital, Queen Elizabeth II Jubilee Hospital, and Princess Alexandra Hospital.

Disclaimer The funder had no involvement in design and will have no role in the collection, management, analysis and interpretation of data; writing of the report; and the decision to submit the report for publication, including no authority over any of these activities.

Competing interests MIC's employer has received on her behalf: an unrestricted research grant from $\mathrm{BD}$, during the conduct of the study. EL's employer has received on her behalf: a consultancy payment from 3M, outside the submitted work. MC's employer has received on her behalf: an unrestricted research grant from BD, during the conduct of the study; unrestricted research grants and/or educational grants from 3M, Baxter, BD, Entrotech, outside the submitted work. NMM's employer has received on her behalf: an unrestricted research grant from BD, 
during the conduct of the study; unrestricted research grants and/or educational grants from BD, Hospira and Adhezion, outside the submitted work; consultancy payments from $B D$ and Hospira, outside the submitted work. MCW's employer has received on her behalf: an unrestricted research grant from $\mathrm{BD}$, during the conduct of the study; unrestricted research grants and/or educational grants from $\mathrm{BD}$, outside the submitted work; consultancy payments from BD, outside the submitted work. PJJC's employer has received on his behalf: an unrestricted research grant from BD, during the conduct of the study. LZ' employer has received on her behalf: an unrestricted research grant from $B D$, during the conduct of the study; unrestricted research grants and/or educational grants from BD, outside the submitted work. GJ's employer has received on his behalf: an unrestricted research grant from BD. CMR's employer has received on her behalf: an unrestricted research grant from $\mathrm{BD}$, during the conduct of the study; unrestricted research grants and/or educational grants from 3M, Adhezion, Angiodynamics, Bard, Baxter, BBraun, BD, Carefusion, Centurion Medical Products, Cook Medical, Entrotech, Flomedical, ICU Medical, Medical Australia, Medtronic, Smiths Medical, Teleflex, outside the submitted work; consultancy payments from 3M, Bard, BBraun, BD, Smiths Medical, ResQDevices, outside the submitted work. No commercial entity had any role in the preparation of this manuscript.

Patient consent Not required.

Ethics approval Royal Brisbane and Women's Hospital Human Research Ethics Committee (EC00172), Griffith University Human Research Ethics Committee (EC00162) and the Royal Perth Hospital Human Research Ethics Committee (EC00270).

Provenance and peer review Not commissioned; externally peer reviewed.

Open Access This is an Open Access article distributed in accordance with the Creative Commons Attribution Non Commercial (CC BY-NC 4.0) license, which permits others to distribute, remix, adapt, build upon this work non-commercially, and license their derivative works on different terms, provided the original work is properly cited and the use is non-commercial. See: http://creativecommons.org/ licenses/by-nc/4.0/

(C) Article author(s) (or their employer(s) unless otherwise stated in the text of the article) 2018. All rights reserved. No commercial use is permitted unless otherwise expressly granted.

\section{REFERENCES}

1. Zingg W, Pittet D. Peripheral venous catheters: an under-evaluated problem. Int J Antimicrob Agents 2009;34:S38-42.

2. Bolton D. Improving peripheral cannulation practice at an NHS Trust. Br J Nurs 2010;19:1346-50.

3. Rickard CM, Webster J, Wallis MC, et al. Routine versus clinically indicated replacement of peripheral intravenous catheters: a randomised controlled equivalence trial. Lancet 2012;380:1066-74.

4. Rickard CM, Marsh N, Webster J, et al. Securing All intraVenous devices Effectively in hospitalised patients--the SAVE trial: study protocol for a multicentre randomised controlled trial. BMJ Open 2015;5:e008689.

5. González López JL, Arribi Vilela A, Fernández del Palacio E, et al. Indwell times, complications and costs of open vs closed safety peripheral intravenous catheters: a randomized study. $J$ Hosp Infect 2014;86:117-26.

6. Loveday HP, Wilson JA, Pratt RJ, et al. epic3: national evidencebased guidelines for preventing healthcare-associated infections in NHS hospitals in England. J Hosp Infect 2014;86:S1-S70.

7. Gorski LA. The 2016 infusion therapy standards of practice. Home Healthc Now 2017;35:10-18.

8. Infusion Nurses Society. Infusion nursing standards of practice. $J$ Infus Nurs 2006;29:S1-92.

9. Beckton, Dickinson, Company. BD Nexiva ${ }^{\mathrm{TM}}$ Closed IV catheter system. https://www.bd.com/en-ca/products/medical-surgicalsystems/infusion-therapy/iv-access/nexiva-closed-iv-cathetersystem (accessed Feb 2018).

10. Sealed Envelope ${ }^{\mathrm{TM}}$. Power calculator for binary outcome superiority trial. https://www.sealedenvelope.com/power/binary-superiority/ (accessed Feb 2018).

11. Helm RE, Klausner JD, Klemperer JD, et al. Accepted but unacceptable: peripheral IV catheter failure. J Infus Nurs 2015;38:189-203.

12. Webster J, Osborne S, Rickard CM, et al. Clinically-indicated replacement versus routine replacement of peripheral venous catheters. Cochrane Database Syst Rev 2015;8:CD007798.

13. Hadaway LC. I.V. infiltration: Not just a peripheral problem. Nursing 1999;29:41-7.

14. Marsh N, Webster J, Mihala G, et al. Devices and dressings to secure peripheral venous catheters to prevent complications. Cochrane Database Syst Rev 2015:CD011070.

15. Centers for Disease Control and Prevention. CDC/NHSN Surveillance definitions for specific types of infections. 2017 https://www.cdc. gov/nhsn/pdfs/pscmanual/17pscnosinfdef_current.pdf (accessed Feb 2018).

16. Griffith University. Griffith randomisation service. https://www151. griffith.edu.au/ (accessed Feb 2018).

17. Harris PA, Taylor R, Thielke $R$, et al. Research electronic data capture (REDCap)--a metadata-driven methodology and workflow process for providing translational research informatics support. $J$ Biomed Inform 2009;42:377-81.

18. Aitken LM, Mardegan KJ. "Thinking aloud": data collection in the natural setting. West J Nurs Res 2000;22:841-53.

19. Herdman M, Gudex C, Lloyd A, et al. Development and preliminary testing of the new five-level version of EQ-5D (EQ-5D-5L). Qual Life Res 2011;20:1727-36.

20. Peipert JD, Beaumont JL, Bode R, et al. Development and validation of the functional assessment of chronic illness therapy treatment satisfaction (FACIT TS) measures. Qual Life Res 2014;23:815-24.

21. Norwood SL. Research strategies for advanced practice nurses. Upper Saddle River, N.J: Prentice Hall Health, 2000.

22. Maki DG, Weise CE, Sarafin HW. A semiquantitative culture method for identifying intravenous-catheter-related infection. N Engl J Med 1977;296:1305-9.

23. StataCorp. Stata Statistical Software: Release 14. College Station, TX: StataCorp LP, 2015.

24. U.S Department of Health and Human Services. Common Terminology Criteria for Adverse Events (CTCAE) Version 4.0. 2010 https://evs.nci.nih.gov/ftp1/CTCAE/CTCAE_4.03_2010-06-14_ QuickReference_5x7.pdf (accessed Feb 2018).

25. International Committee of Medical Journal Editors. Preparing for submission secondary preparing for submission. $2017 \mathrm{http}: / /$ www.icmje.org/recommendations/browse/manuscript-preparation/ preparing-for-submission.html (accessed Feb 2018). 